\title{
The Position Monitoring System of Automated Guided Vehicle (AGV) In The Industrial Production Process
}

\author{
Yusi Duwi Satriyo ${ }^{1+}$, Angga Rusdinar ${ }^{2}$ and Ig. Prasetya Dwi Wibawa ${ }^{3}$ \\ School of Electrical Engineering, Telkom University, 40257 Bandung Indonesia
}

\begin{abstract}
Robots are needed to enhance the competitiveness and sustainability of the company. Automated Guided Vehicle (AGV) is one of many varieties of industrial robots carrying loads to where we want to be. The wide areas within industrial company inflict the monitoring of production processes to be inadequate. This research discusses the design and implementation of the position monitoring system of AGV. The monitoring system applies wireless communication using Xbee Module. There are two systems: the AGV and user system. The AGV system discussed about the movement control using Fuzzy Inference System (FIS) while the user system discussed about data processing obtained from the AGV system became position coordinate. The results showed the movement of AGV that can be monitored well.
\end{abstract}

Keywords: Automated Guided Vehicle, monitoring system, xbee, fuzzy inference system.

\section{Introduction}

Robotics technology plays a highly important role in the industrial revolution. The utilization of robots has been applied in almost all production sectors of the factory, started from processing to packaging. It is used to improve the efficiency and the quality of products. Hence, it is important for the company to enhance the competitiveness and sustainability of enterprises in a dynamic environment, information, technology, and industrial automation [1]. One of many types of robots employed in the company AGV. It is industrial robot used to carry the loads from one point to another point, both raw materials and final products. It can be like towing vehicle, semiautomatic pallet trucks, or unit load carriers. In several usages, it is programmed to move towards a destination using automatic navigation. Therefore, the operator is assigned to monitor and control it from a distance. AGV is controlled on a central computer containing information about the entire system within the company and directing AGV to its destination [2-3]. Numerous studies of AGV have been performed. There are a lot of aspects studied of them. They contain about control, monitoring, and communication systems of AGV [4-11]. Many studies on algorithms and methods on routing or traffic control systems from AGV have been proposed. Silvirianti et al. presented the movement control system using Fuzzy integrated with PID method [4], while Rusdinar et. al presented the utilization of Kalman Filter and artificial landmark on ceiling applied on AGV [5]. Tavares et al. [6] analysed that there are several aspects that are quite complex when AGV is implemented in the industrial companies. Those are the reasons why the research on communication systems from AGV is less addressed. Tavares also mentioned several researches of AGV. Most of them [7-10] do not present its communication system but focused on the traffic control system.

AGV has automatic navigation in which being controlled and monitored from far away. The damage occurred to AGV being stopped anywhere unbeknown by the operator. We argued that handling for AGV is going to take longer time that can hamper the production process involving in a loss of quality. The quality

\footnotetext{
+ Corresponding author. Tel.: + 6281232358903.

E-mail address: yusisatriyo@gmail.com.
} 
of manufacturing production is not able to succeed without an effective monitoring and control system in the industry, because it is a key requirement industrial production process [12].

In this research, we proposed monitoring system from the research of AGV performed by Sakir et al. in 2017 [11] based on wireless communication. There were two areas performed: AGV system and the user system. AGV system discussed the data processing of the wheel velocity using FIS. The output of AGV system is a linear velocity of the right and left wheels in which they are the data required by the user as input. Prior to user acceptance, data is transmitted over a wireless communication network. The information accepted was processed into coordinates of positions that was presented on the display as monitoring. The experiment results show that AGV movement could be monitored on the display well.

\section{The Development Platform}

\subsection{Automated Guided Vehicle (AGV)}

AGV developed in this research is performed by Sakir et al [11] in 2017. They performed movement controlled AGV using FIS. FIS was implemented because FIS is one of the popular methods in the field of control systems and robots. The AGV System consisted of a slave and master as shown by Fig. 1(b). The inputs of the system were line follower sensor as navigation and encoder installed on AGV. There were two FIS implemented in this research, viz., FIS I and FIS II. FIS I had the function to set the speed of AGV's wheel based on the line sensor (Slave) and the output of FIS I became the reference of FIS II to control the actual speed of AGV in Pulse Width Modulation (PWM) to get the stable speed (Master). The stable speed of AGV was read by the encoder. The data obtained from AGV system was actual speed of the right and left wheel. The information was delivered to user.

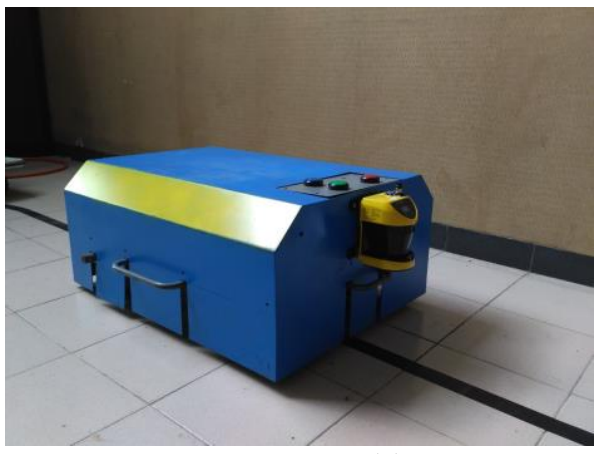

(a)

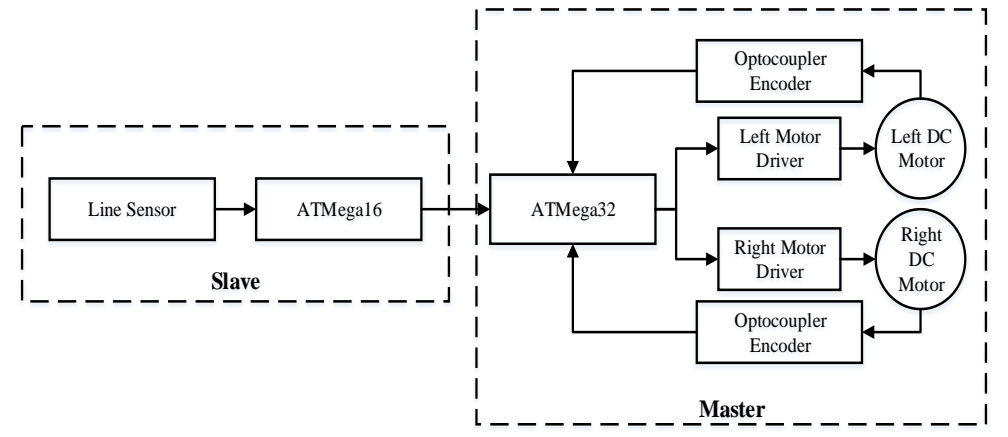

(b)

Fig. 1: The AGV's (a) overview and (b) block diagram system

\subsection{The user}

The user received the information from AGV system via wireless communication using Xbee Zigbee. It was used for the transceiver and receiver data and information. Xbee uses IEEE 802.15.4 protocol and operates within the new standard frequency range, $2.4 \mathrm{GHz}$ in which is better than 802.11 protocol. This protocol has advantages that is low-cost and minimal power requirements [13,14]. In this experiment we used two Xbee. Fig. 2 shows that the first Xbee became the transmitter data obtained from AGV and the second Xbee served as the receiver of information sent and to be processed on personal computer for getting the new data to show on the display.

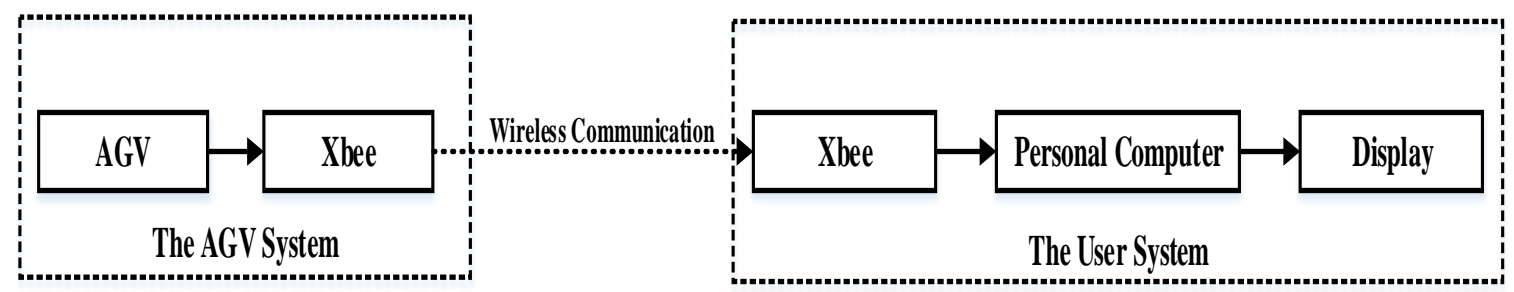

Fig. 2: The communication system of AGV and user 


\section{The System Model Configuration}

\subsection{System configuration}

The data retrieval process of AGV were started from encoders. The encoders were used to measure the rotation of a front right and left wheel. The information obtained by the encoders are the digital data that must be processed into the pulses. The microcontroller received the pulses and defined them to be the encoder values. The values were calculated to be the velocity of the right and left wheels [4]. The data processing is shown by Fig. 3 (a). The information already obtained is going to be delivered to user via wireless using Xbee.

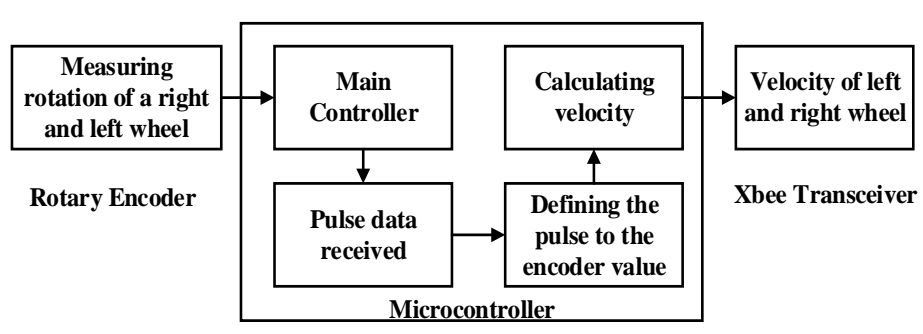

(a)

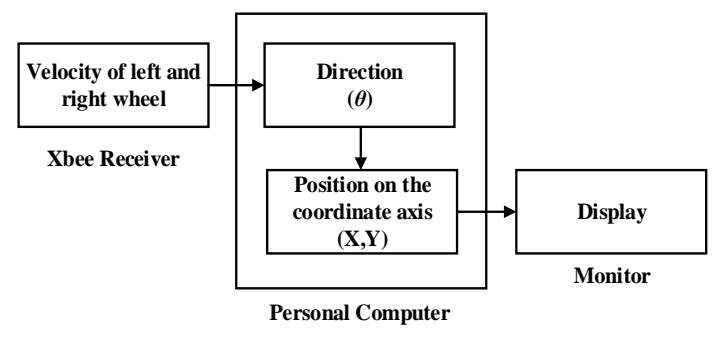

(b)

Fig. 3: The system configuration of (a) AGV and (b) user.

Fig. 3 (b) shows data processing after receiving the information from AGV. The data is the velocity of the front wheels. Personal computer calculated it into the coordinate position using Matlab. The coordinate position determines the AGV movement represented by (x,y) points and displayed in a Cartesian coordinate. The AGV movement can be monitored as a moving point. It is obtained from the velocity computed into the direction. The direction is angular displacement of AGV movement which is calculated from the linear velocity of the front wheels.

\subsection{Movement model}

The formation of the control system model on AGV is based on the coordinate point obtained from the calculation of the rotary encoder. We used several variables that was configured with the sensor. The variables were derived based on the distance between the follower robot and the guiding robot. They were $\omega, \alpha, E$, and $v$.

The angular velocity on the right or left front wheel can be defined by

$$
\begin{gathered}
\omega_{(L, R)}=\frac{\mathrm{rad}}{\mathrm{sec}} \\
\alpha=\frac{360^{\circ}}{n}
\end{gathered}
$$

where rad and sec are the angle of two adjacent slits and the number of slits in the encoder.

$$
\omega_{(L, R)}=\frac{E_{(L, R)} \times \alpha \times \frac{\pi}{180^{\circ}}}{\text { time }}
$$

From Eq. 1 and Eq. 2, we can find the angular velocity that can be converted directly in standard of $\mathrm{rad} / \mathrm{sec}$, while $\mathrm{E}_{(\mathrm{L}, \mathrm{R})}$ is the right or left encoder value (digital value).

The controlled parameter for the AGV movement are the velocity of the left and right of AGV's wheels. The AGV velocity and angular velocity that can be defined by

$$
\begin{gathered}
v_{(L, R)}=\omega_{(L, R)} \times r \\
v=\frac{v_{L}+v_{R}}{2} \\
\omega=\frac{v_{L}-v_{R}}{l}
\end{gathered}
$$


where $v_{L}$ and $v_{R}$ are the variable of the linear velocity of the left and right AGV's wheels, and the width of the vehicle is $l$. Eq. 4 determine the linear velocity of AGV, where $\omega$ is the angular velocity of AGV's wheel, and $r$ is radius of wheel. From Eq. 1 till Eq. 6, we can define the AGV movement [5,6] as follows:

$$
\left[\begin{array}{l}
x \\
y \\
\theta
\end{array}\right]=\left[\begin{array}{l}
x_{0} \\
y_{0} \\
\theta_{0}
\end{array}\right]+\left[\begin{array}{l}
v \cos \left(\theta_{0}+\omega\right) \\
v \sin \left(\theta_{0}+\omega\right) \\
\left(\theta_{0}+\omega\right)
\end{array}\right]
$$

where the AGV position and the direction uses variables $x, y$ and $\theta$ respectively; while $x_{0}, y_{0}$ and $\theta_{0}$ is the previous or starting point and direction of AGV. $v$ presents as the AGV velocity and $\omega$ is angular velocity of AGV.

\section{Experiment Result}

The experiments were performed. There were two areas researched: the AGV system and the user system. The AGV system was an area in which studied the data process obtained from AGV supposed to deliver to the user. The user system is an area in which monitoring process will be shown after receiving the information from the AGV system.

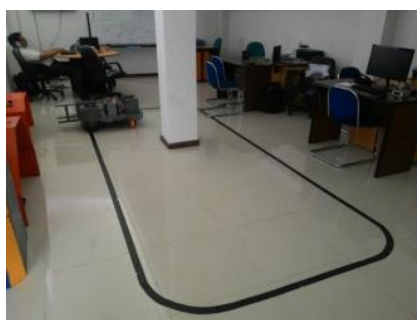

(a)

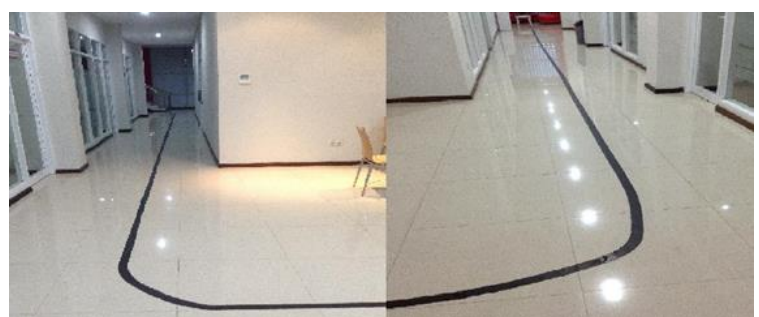

(b)

Fig 4: The AGV trajectory (a) small and (b) large scale.

The first experiment was done by implementing AGV in the distinctive trajectories crossed by the robot (The AGV System). Fig. 4 shows the trajectories. They had two scales: large and small. The large scale had a total path along the 47.6 meters, while the small scale had a length, 11 meters. AGV followed the track line according to the data obtained from photodiode sensors. The differences of the speed between the right and left wheel caused AGV turned. AGV turned left when the right wheel had faster speed than left wheel vice versa.

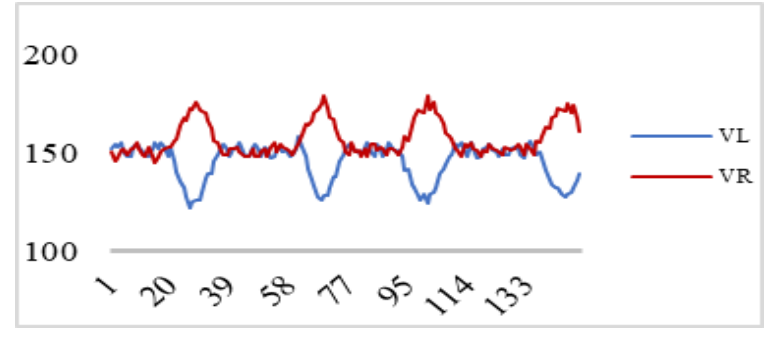

(a)

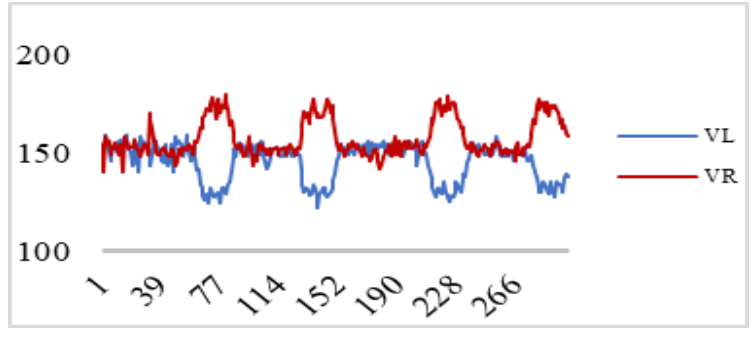

(b)

Fig. 5: Output FIS-1 for (a) small and (b) large scale.

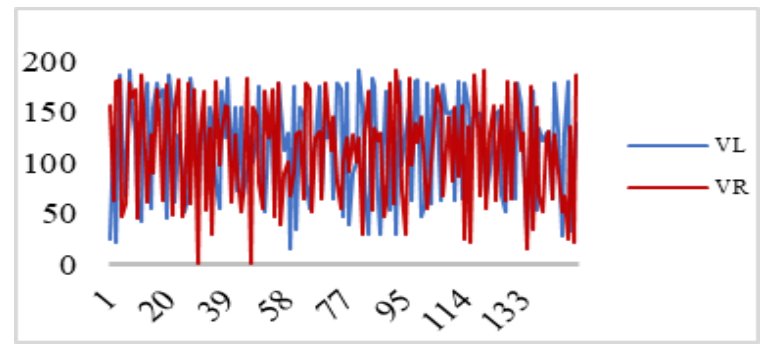

(a)

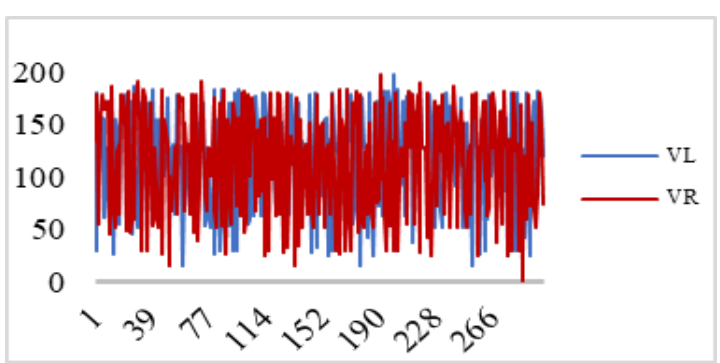

(b)

Fig. 5: Output FIS-2 for (a) small and (b) large scale. 
The graphic of Fig. 5 shows the results of FIS-2. The results were the speed of AGV in PWM. The actual speed of AGV adjusted to the output of FIS, then obtaining the stable speed of AGV. The actual speed of AGV obtained was delivered to the user by wireless communication.

Before doing the data processing to get the coordinate position, we needed to test the communication system. The communication test of Xbee Module was done by transmitting the right and left wheel speed on the receiver (user) so it could be concluded whether there was interference or not when the delivery process. This test was undertaken within less than 150 meters. All data transmitted were received by the receiver properly without any interference as shown by Fig. 6 .

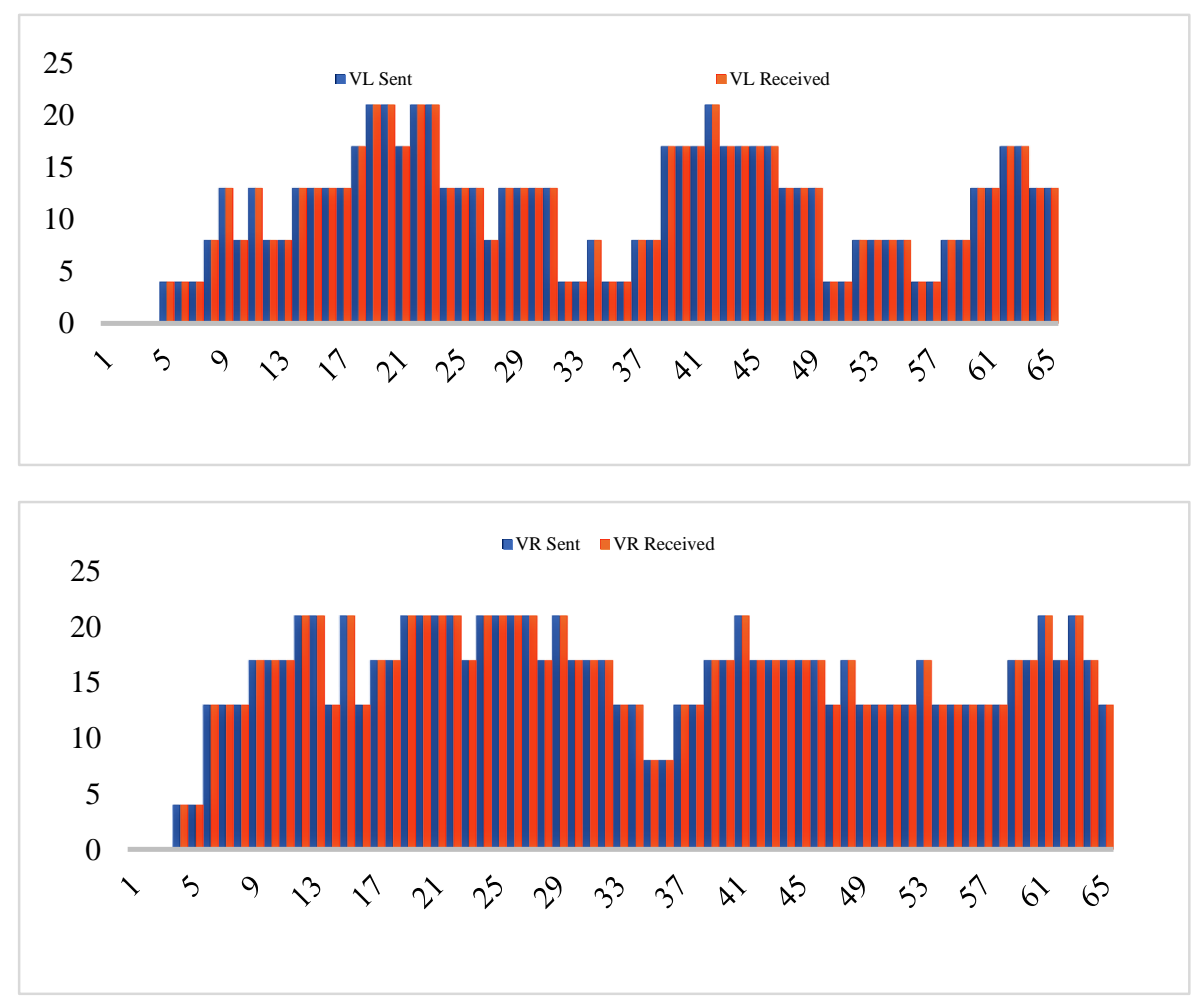

Fig. 6: Transmitting and receiving data (a) left and (b) right wheel.

The user received data from AGV and processed them becoming the coordinate position. The first experiment was performed in the user that was data processing by receiving actual speed of AGV in the straight track. The movement of AGV could be good monitored based on mapping of coordinate position estimation as shown by Fig. 6 (a). This test was performed to determine the position of robots based on $v_{\mathrm{L}}$ and $\mathrm{v}_{\mathrm{R}}$ received with the initial position of $\mathrm{x}$ and $\mathrm{y}$ are coordinated. The calculation results among $\mathrm{x}, \mathrm{y}$, and $\theta$ will be accurate in case the data $\mathrm{v}_{\mathrm{L}}$ and $\mathrm{v}_{\mathrm{R}}$ received are correct. Data transmitted for one round has no error as shown by Fig. 6 (b).
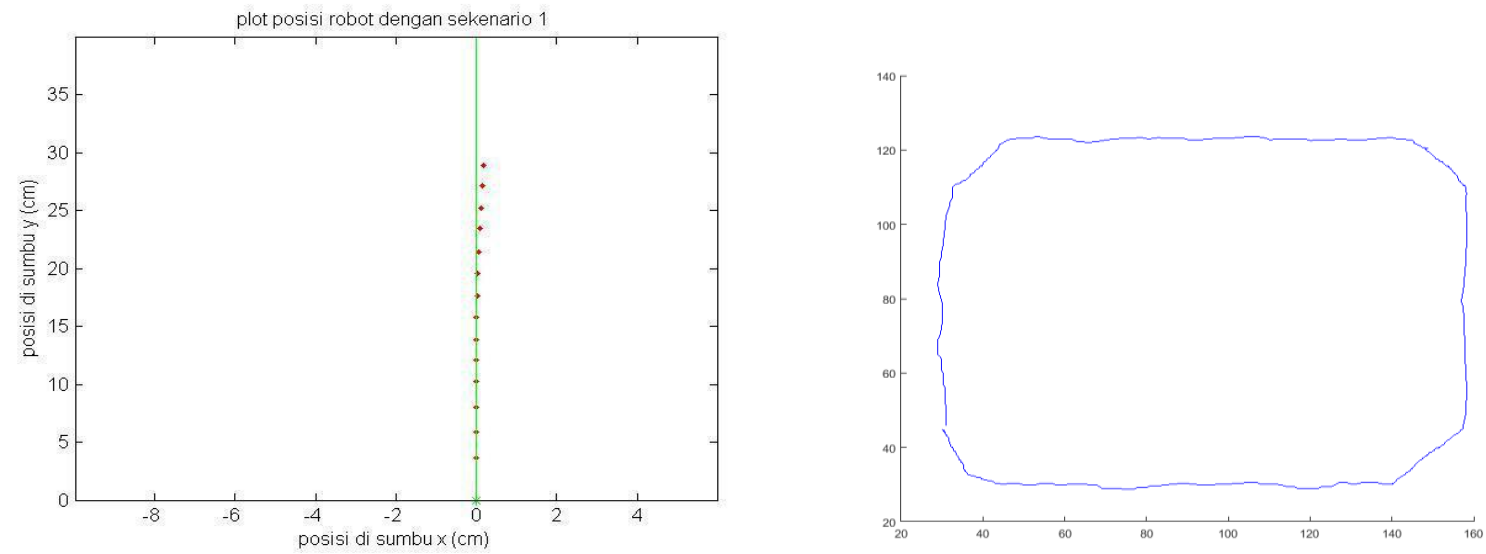

Fig. 6: The AGV movement in (a) straight and (b) round track. 


\section{Conclusion}

This paper presents monitoring system of AGV using FIS as movement control algorithm. The fuzzy system helped to control and maintain the AGV speed. The research performed that the AGV movement can follow the line sensor well. The movement of AGV can be monitored by calculating speed data obtained and processed to be coordinate position but it is considered less accurate because there is interference in turn path, while for communication system can send and receive data without any noise.

\section{Acknowledgement}

The research presented was supported by Information and Autonomous Control System (INACOS) Laboratory of School of Electrical Engineering of Telkom University and funded by Ministry of Research Technology and higher Education of Republic of Indonesia (RISTEK DIKTI) trough Industrial Technology Development Program (PPTI) 2018.

\section{References}

[1] R. W. C. Martins, "A modular architectore for the control of fms (uma arquitetura modular para controle de fms)," Master's thesis, Departament of Computing, Universidade Federal de Sao Carlos, 2005.

[2] Ullrich, Günter Automated Guided Vehicle Systems: A Primer with Practical Applications Oberheimbach, Germany.

[3] Deborah A. Davis, "Modeling AGV System", Proceedings of the 19\&F Winter Simulation Conference J. Wilson, J. Henriksen, S. Roberts (eds.), pp 568-574.

[4] Silvirianti, Riesa Krisna A.S., Angga Rusdinar, Sigit Yuwono4, R. Nugraha, "Speed Control System Design Using Fuzzy-PID for Load Variation of Automated Guided Vehicle (AGV)", 2nd International Conference on Frontiers of Sensors Technologies, 2017, pp 426-430.

[5] Angga Rusdinar, Jungmin Kim, Junha Lee, Sungshin Kim, "Implementation of real-time positioning system using extended Kalman filter and artificial landmark on ceiling", Journal of Mechanical Science and Technology 26 (3) (2012) 949-958.

[6] Tavares, Dalton Matsuo, and Bachega, Stella Jacyszyn , "Proposal for an AGV Communication System Using a Cellbot Framework", 10th IEEE/IAS International Conference on Industry Applications, 2012.

[7] S. Shoval, I. Zeitoun, and E. Lenz, "Implementation of A Kalman Filter in Positioning for Autonomous Vehicles, and Its Sensitivity to The Process Parameters," The International Journal of Advanced Manufacturing Technology, vol. 13, pp. 738-746, 1997.

[8] M. Watanabe, M. Furukawa, and Y. Kakazu, "Intelligent AGV Driving Toward An Autonomous Decentralized Manufacturing System," Robotics and Computer Integrated Manufacturing, vol. 17, pp. 57-64, 2001.

[9] S. K. Kim, B. D. Chung, and M. Jae, “A Design for A Tandem AGVs with Multi-Load AGVs," The International Journal of Advanced Manufacturing Technology, vol. 22, pp. 744-752, 2003.

[10] A. Salehipour, H. Kazemipoor, and L. M. Naeini, "Locating Workstations in Tandem Automated Guided Vehicle Systems,” The International Journal of Advanced Manufacturing Technology, vol. 52, pp. 321-328, 2011.

[11] Riesa Krisna Astuti Sakir, Angga Rusdinar, Sigit Yuwono, Agung Surya Wibowo, Silvirianti, Nadia Tri Jayanti, "Movement Control Algorithm of Weighted Automated Guided Vehicle Using Fuzzy Inference System", 2nd International Conference on Control and Robotics Engineering, 2017, pp 135-139.

[12] Krist V. Gernaey, Jarka Glassey, Sigurd Skogestad, Stefan KräMer, Andreas Weiß, Sebastian Engell, Efstratios N. Pistikopoulos, David B. Cameron, "Process Monitoring", Proceeding of Ullmann's Encyclopedia of Industrial Chemistry Academy.

[13] Muhammad Umer Shahzad,Abdul Qayyum Khan, SM.Rameez Bukhari,Aqil Aslam,M.Orang Zaib, "Wireless Control Robot Using Xbee Module with Multiple Sensor Acknowledgment on HMI”, 2017.

[14] Sam Shue, Lauren E. Johnsony, James M. Conrad, "Utilization of XBee ZigBee Modules and MATLAB for RSSI Localization Applications", SoutheastCon 2017. 